Kompass

Autoimmun

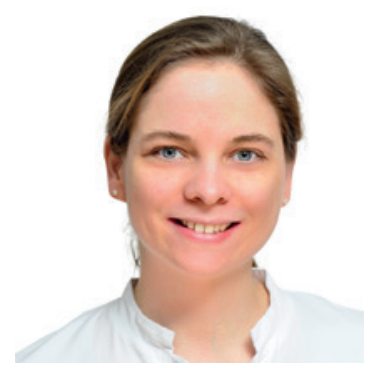

\title{
Wie beeinflussen Nierenschäden unter Checkpointinhibitortherapie die Prognose?
}

Sibylle von Vietinghoff

Universitätsklinikum Bonn, Sektion für Nephrologie, Medizinische Klinik und Poliklinik I, Bonn, Deutschland

Abstract aus Koks MS, Ocak G, Suelmann BBM, et al.: Immune checkpoint inhibitor-associated acute kidney injury and mortality: An observational study. PLoS One 2021;16:e0252978.

\begin{abstract}
Background: Immune checkpoint inhibitors, approved for the treatment of various types of cancer, are known to cause a unique spectrum of side effects, including acute kidney injury (AKI). The aim of this study was to describe the incidence, risk factors, renal outcomes, and mortality of AKI in patients receiving checkpoint inhibitors.

Methods: Patients receiving checkpoint inhibitors between January 2013 and May 2020 at the University Medical Center Utrecht, the Netherlands, were identified using the Utrecht Patient Oriented Database. AKI was defined as an increase in serum creatinine of $\geq 1.5$ times the baseline value, based on the Kidney Disease: Improving Global Outcomes criteria. Cox proportional hazard regression analysis was used to assess risk factors for AKI and to evaluate the relationship between AKI and mortality. Persistent renal dysfunction was diagnosed in AKI patients with a final serum creatinine measurement of $>1.3$ times the baseline value.
\end{abstract}

Results: Among 676 patients receiving checkpoint inhibitors, the overall incidence of AKI was 14.2\%. Baseline variables independently associated with AKI were a gynecologic malignancy, monotherapy with ipilimumab, and the use of a diuretic, angiotensin-converting enzyme inhibitor or angiotensin-receptor blocker, or proton pump inhibitor at baseline. AKI was checkpoint inhibitor-associated in one third of all patients with AKI. Checkpoint inhibitor-associated AKI was mostly low-grade, occurred a median of 15 weeks after checkpoint inhibitor initiation, and resulted in persistent renal dysfunction in approximately $40 \%$ of the patients. Patients with all-cause AKI had a twofold increased mortality risk, but checkpoint inhibitor-associated AKI was not associated with increased mortality.

Conclusions: In this study, patients receiving checkpoint inhibitors frequently developed AKI due to various etiologies. AKI directly related to the effect of checkpoint inhibitor toxicity did not increase mortality. However, AKI not related to the effect of checkpoint inhibitor toxicity was associated with increased mortality. 


\section{Transfer in die Praxis}

\section{Akute Nierenschädigung in der Tumortherapie}

Ein akuter Nierenschaden (AKI) tritt bei Patienten mit Tumorerkrankungen häufig auf [1]. Dies umfasst direkte Schäden durch den Tumor ebenso wie Nebenwirkungen der Therapie. Insgesamt ist ein AKI, wie bei vielen anderen Erkrankungen auch, mit einer schlechteren Prognose assoziiert.

Zum medikationsinduzierten AKI gehören die direkte Tubulo- und Glomerulotoxizität vieler Chemotherapeutika und indirekte Effekte, zum Beispiel über ein Tumorlysesyndrom. Immuntherapien haben die Prognose vieler Tumorerkrankungen dramatisch verbessert, aber diese Schädigungsmechanismen auch um neue Autoimmunphänomene erweitert. Die Gruppe der Checkpointinhibitoren verhindert, dass autoreaktive T-Zellen in ihrer Aktivität eingeschränkt werden. Dies ist als antitumoraler Effekt gewünscht, kann aber auch bestehende oder neue autoreaktive T-Zellen desinhibieren. Die Zielmoleküle sind dabei aktuell inhibitorische Moleküle auf der T-Zell-Oberfläche wie CTLA-4 (cytotoxic T lymphocyte-associated protein 4) und PD-1 (programmed cell death 1) oder sein Ligand PD-L1. Autoimmunphänomene wurden in verschiedenen Organen beschrieben. Häufig ist die Haut oder die Hypophyse betroffen [2]. Auch akute Nierenversagen werden vermehrt beobachtet.

\section{Formen des AKI bei Checkpointinhibition}

Eine aktuelle Metaanalyse randomisierter Studien, in denen das Auftreten von AKI unter PD-1- und PD-L1-Inhibitoren in soliden Tumoren in Studien mit insgesamt über 15000 Patienten zusammengefasst wurde, zeigt ein signifikant erhöhtes Risiko für einen Anstieg des Serumkreatinin-Levels oder eines formalen AKI mit einem relativen Risiko von 2,77 [3].

Frühere Kohorten mit histologischer Sicherung der Nierenschädigung zeigen in vielen Fällen eine interstitielle Nephritis [4, 5]. Aber auch Glomerulonephritiden wurden beobachtet.

\section{Studienkohorte}

Die hier diskutierte Arbeit adressiert Risikofaktoren für einen AKI. Es handelt sich um eine longitudinale Datensammlung an der Universität Utrecht von 2013 bis 2020. Insgesamt wurden die Daten von 676 Patienten analysiert, die einen Checkpointinhibitor erhielten. Davon erlitten 14,2\% einen AKI. Die Arbeit versucht, Unterschiede innerhalb der Checkpointinhibitor-behandelten Patienten herauszuarbeiten. Ein Vergleich, ob dieselben Faktoren auch bei anderen Gruppen mit einem AKI assoziiert sind, war nicht Teil der Untersuchung.

Insgesamt waren die meisten AKI $(n=76)$ im Stadium 1, eine Dialysebehandlung wurde von keinem Patienten benötigt. In der hier beschriebenen Kohorte wurden nur 12,5\% der AKI-Patienten von einem Nephrologen gesehen, immerhin etwas über 56 Patienten erhielten eine Urinanalyse. Nur 1 Patient erhielt eine Nierenbiopsie. In insgesamt etwa 28\% der Fälle wurde von einer tubulointerstitiellen Nephritis ausgegangen. Dagegen war in gut der Hälfte der Fälle von einem prärenalen Problem auszugehen, auch eine postrenale Ursache im Sinne einer Obstruktion fand sich mit 7,3\% relativ häufig. Andere Ursachen wie Pyelonephritiden oder Cast-Nephropathie waren sehr selten.

Von den 32 Patienten mit möglichem oder wahrscheinlichem AKI durch Checkpointinhibitorblockade war der Anteil des milden AKI noch einmal höher als in der Gesamtkohorte: über 80\%. 11 Patienten erhielten Steroide, bei 44\% wurde die Therapie unterbrochen und größtenteils nicht wiederaufgenommen.

Die Risiken für AKI wurden in der Gesamtkohorte analysiert, d.h. diese Analyse umfasst auch alle anderen, insbesondere auch die hämodynamischen Ursachen. Als Risikofaktoren fanden sich gynäkologische Tumoren und die Gabe des CTLA-4-Blockers Ipilimumab. Eine umfangreiche Adjustierung (Alter, Geschlecht, Malignomtyp, vorangegangene Ras-Blockade, Protonenpumpenhemmer (PPI), nichtsteroidale Antirheumatika (NSAID), vorangegangene Chemotherapie, Morbiditätsindex) geht sicher an die Grenze des mit dem vorliegenden Datensatz Leistbaren. Bei manchen Komedikationen (Angiotensin-Converting-Enzyme (ACE)-Hemmer, Diuretika), die das relative Risiko für ein AKI erhöhten, stellt sich die Frage, ob diese Basismedikation nicht auch auf ein chronisches Nierenversagen (CKD) hindeutet, sodass ein bedeutender Confounder bestünde. Auch PPI erhöhten das Risiko, dies ist aus der Literatur auch in anderen klinischen Konstellationen [1] und in einer vergleichbaren Checkpointinhibitorarbeit [6] ebenfalls auffällig gewesen.

Daten zur Nierenfunktion vor dem Ereignis fehlen. In vorangegangenen Arbeiten in einem anderen Zentrum war ein CKD aber kein Prädiktor für einen Checkpointinhibitor-vermittelten AKI [6]. Die Mortalität stieg nach multipler Adjustierung mit dem Auftreten von AKI, aber nicht dem, das klinisch auf eine Checkpointinhibition zurückgeführt wurde.

\section{Fazit}

Diese retrospektive Analyse kann den Eindruck der «Real-World»Daten des AKI unter PD-1-Blockade ein wenig erweitern, letztlich sind die Patientenzahlen trotz der langfristigen Datenerhebung klein. Ihre Ergebnisse stimmen insgesamt mit größeren und mit ausgewählten Kontrollen gematchten Kohorten überein [7, 8].

\section{Disclosure Statement}

Keine für diese Arbeit relevanten Interessenkonflikte.

\section{Literatur}

1 Rosner MH, et al. N Engl J Med. 2017;376:1770-1781.

2 Bluestone JA, et al. N Engl J Med. 2020;383:1156-1166.

3 Li H, et al. Invest New Drugs. 2021;39:860-870.

4 Cortazar FB, et al. Kidney Int. 2016;90:638-647.

5 Mamlouk O, et al. J Immunother Cancer. 2019;7:2

6 Seethapathy H, et al. Clin J Am Soc Nephrol. 2019;14:1692-1700.

7 Cortazar FB, et al. J Am Soc Nephrol. 2020;31:435-446.

8 Gupta S, et al. J Immunother Cancer. 2021;9:e003467.

Korrespondenz an:

Prof. Dr. Sibylle von Vietinghoff, Sibylle.von_Vietinghoff@ukbonn.de 